\title{
Towards an Estimation of Acoustic Impedance from Multiple Ultrasound Images
}

\author{
Christian Wachinger ${ }^{1}$, Ramtin Shams ${ }^{2}$, Nassir Navab ${ }^{1}$ \\ ${ }^{1}$ Computer Aided Medical Procedures (CAMP), Technische Universität München \\ ${ }^{2}$ Research School of Information Sciences and Eng., The Australian National Univ. \\ wachinge@in.tum.de
}

\begin{abstract}
Reflection of sound waves, due to acoustic impedance mismatch at the interface of two media, is the principal physical property which allows visualization with ultrasound. In this paper, we investigate reconstruction of the acoustic impedance from ultrasound images for the first time. Similar to spatial compounding, we combine multiple images to improve the estimation. We use phase information to determine regions of high reflection from an ultrasound image. We model the physical imaging process with an emphasis on the reflection of sound waves. The model is used in computing the acoustic impedance (up to a scale) from areas of high reflectivity. The acoustic impedance image can either be directly visualized or be used in simulation of ultrasound images from an arbitrary point of view. The experiments performed on in-vitro and in-vivo data show promising results.
\end{abstract}

\section{Introduction}

Ultrasound (US) has many advantages in comparison to other imaging modalities which has lead to its widespread use in clinical practice; it is (i) harmless at low power, (ii) portable, (iii) a real-time modality, and (iv) most importantly, cost effective. The recent introduction of $2 \mathrm{D}$ array US transducers in the market makes further applications possible, due to the instantaneous acquisition of ultrasound volumes. Furthermore, the next generation of transducers with capacitive micromachined ultrasound transducer (CMUT) technology offers superior and efficient volumetric imaging at a lower cost. However, ultrasound has a number of disadvantages including: (i) a limited field-of-view (FOV), (ii) occlusions behind structures with high acoustic impedance, and (iii) a low singal-to-noise ratio (SNR). Spatial compounding of several views, acquired from different positions, helps to reduce these shortcomings.

The prerequisite for spatial compounding is to know the relative positions of the acquired images. This can either be obtained by tracking the ultrasound transducer or by image registration. When working with 2D US images compounding from different positions poses a problem, because all the scans have to be in one plane. Therefore, multi-angle compounding with beam steering is typically performed, where the probe remains fixed [1]. Moving to 3D imaging, 
compounding from different positions offers much more flexibility, inspiring several groups to work on this subject e.g. [2,3]. In the following, we assume that we know the alignment of the images, and focus on how to combine their intensity information. As we will discuss later in this report, combining ultrasound images is non-trivial due to the highly view-dependent nature of the ultrasound. We will introduce a novel approach, which is based on the estimation of the acoustic impedance of the imaged scene [4]. From each image, we will reconstruct an acoustic impedance image, which we subsequently average to get an estimation for the whole imaged area. These images can either be directly presented to the physician or can be used in simulation of ultrasound images from an arbitrary point of view.

\section{Acoustic impedance estimation}

Core to our method is the estimation of the acoustic impedance of the region depicted in the ultrasound image. As we will see, acoustic impedance images are related to CT attenuation values expressed in Hounsfield units and no longer exhibit view-dependent artifacts and emphasized interface boundaries as in ultrasound images. Having the acoustic impedance images $z_{i}$ from all views, the creation of a global acoustic impedance image $z$ for the whole imaging scenario is possible.

We first reduce speckle in the images by median filtering them, and further identify regions of high reflectivity by calculating the local phase. Third, we use these regions to reconstruct the impedance for each image, and finally we find the global impedance estimation by averaging acoustic images obtained from each ultrasound image.

\subsection{Phase calculation}

Essential for the acoustic impedance estimation is the identification of regions with high reflectivity, indicating a change in acoustic impedance. We use phase information for this purpose because it provides us with structural information independent of the brightness and contrast [2]. For 1-D signals the phase is constructed from the original signal and its Hilbert transform. There are different approaches to extend this concept to $N$-D. Here we will use the recently introduced monogenic signal approach [5]. It uses a generalization of the Hilbert transform, the Riesz transform, to calculate phase information in $N$-D. The image is filtered by $N$ filters, which are given in the Fourier domain by

$$
R_{i}\left(f_{1}, \ldots, f_{N}\right)=\frac{f_{i}}{\sqrt{\sum_{j=1}^{N} f_{j}^{2}}}
$$

with $f_{1}, \ldots, f_{N}$ the Fourier domain coordinates. We follow [2] in applying logGabor filters prior to the calculation of the monogenic signal of the image to extract frequency and spatial localization. The monogenic signal provides us 
with information about the phase and orientation of each pixel (Fig. 1). We threshold the phase image to get a mask (Fig. 1) to extract the reflectivity part from the ultrasound image.

\subsection{Acoustic impedance calculation}

We estimate for each US image $u_{i}$ an acoustic impedance $z_{i}$

$$
\arg \min _{z_{i}}\left(u_{i}(\mathbf{x})-\frac{\log \left(1+a \cdot(\cos \varphi(\mathbf{x}))^{m} \cdot \rho_{z_{i}}(\mathbf{x})\right)}{\log (1+a)}\right)^{2}
$$

with $a$ paramterizing the log-compression, $\varphi(\mathbf{x})$ the incidence angle at position $\mathbf{x}, m$ modeling the heterogeneity of the interface, and the reflection coefficient

$$
\rho_{z}(\mathbf{x})=\left(\frac{z(\mathbf{x})-z(\mathbf{x}-\Delta \mathbf{d})}{z(\mathbf{x})+z(\mathbf{x}-\Delta \mathbf{d})}\right)^{2} .
$$

In order to perform the optimization, we make the assumption that the incidence angle for the impedance and ultrasound image are roughly the same

$$
\cos \varphi(x)=\left|\mathbf{d} \cdot \frac{\nabla z(\mathbf{x})}{|\nabla z(\mathbf{x})|}\right| \approx\left|\mathbf{d} \cdot \frac{\nabla u(\mathbf{x})}{|\nabla u(\mathbf{x})|}\right| .
$$

Since the orientation of the interfaces in impedance and ultrasound image should be the same, this approximation is reasonable.

The only term in Equation (2) that is still dependent on the acoustic impedance is the reflection coefficient $\rho_{z_{i}}(\mathbf{x})$. Since this term is recursively defined, depending on the previous estimation $z(\mathbf{x}-\Delta \mathbf{d})$, we need an initial value to make the calculation along the scanline. When taking e.g. acquisitions in a water bath, we can directly use the acoustic impedance of water. But it is not always possible to acquire a proper initialization and as such, in general, a reconstruction up to a scale is possible. This is sufficient for visualization and US simulation. In the future, further information from the US image such as tissue estimation from RF data or speckle could be integrated to make the estimation more precise.

Having the acoustic estimates $z_{i}$, we get the global acoustic impedance image $z$ by calculating the mean.

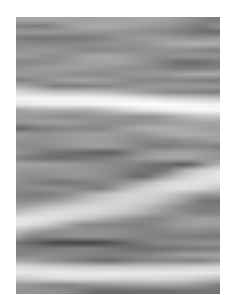

(a) Original

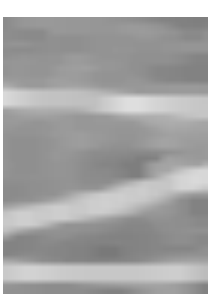

(b) Filtered

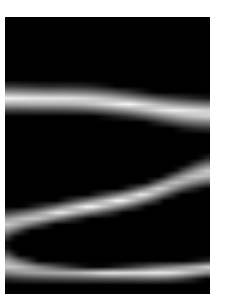

(c) Phase

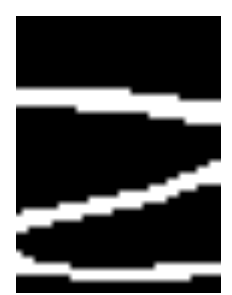

(d) Thres.

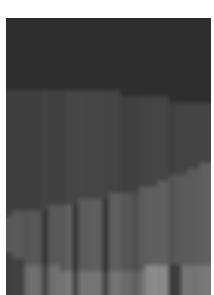

(e) Impedance

Fig. 1. Processing steps for acoustic impedance estimation of simulated ultrasound 


\section{Visualization}

Once the global acoustic impedance image $z$ is estimated, we have to find ways to visualize it for the physician. One possibility would be to directly present the acoustic impedance image, but this may be of limited clinical value, because physicians are not used to these images and may have problems interpreting them. A better way may be to create artificial ultrasound views [6], see Figure $3(\mathrm{~h})$. The simulation of US from the acoustic impedance image is feasible because it is the acoustic impedance that determines the structure of the US images. It has the advantage, that US views can be simulated that were initially not recorded, and from positions that are physically not possible.

\section{Results}

We perform experiments on simulations of ultrasound from CT [6] producing realistic test images. The images are acquired from different viewing angles varying from $-20^{\circ}$ to $20^{\circ}$ to create a realistic spatial compounding scenario. We show as an example the processing steps for the estimation of the simulated US images acquired at $0^{\circ}$ in Figure 1 , with a wavelength of $60 \mathrm{~mm}, a=83$, and $m=0.1$. After doing the same with the four remaining images and mapping them back to the reference frame at $0^{\circ}$, see Figures $3(\mathrm{a})-3(\mathrm{e})$, we can calculate the global acoustic impedance image, see Figure 3(f). It is the average of the separate estimations and one clearly sees the improved quality. When comparing it to the original CT image, which can to some respect be seen as ground truth, the good quality of the reconstruction becomes apparent. Finally, we use the global impedance image to simulate an ultrasound image, see Figure 3(h), with the same method we originally used to simulate the US images from CT.

\section{Conclusion}

We have presented a method to estimate acoustic impedance from multiple ultrasound images. The key to the acoustic impedance calculation is to have a model from the physical imaging process to be able to analyze US images. We have presented such a model and adapted it to the specific needs of acoustic impedance estimation, where we focused on modeling reflections. This allows us to estimate the impedance values at tissue interfaces. We proposed a phasebased image analysis to extract regions of high reflection from the image. Similar to spatial compounding, we average several images to get a robust estimation of the global acoustic impedance. Our results show that acoustic impedance estimation is feasible. It would, however, be helpful to integrate further data in the estimation process coming from RF, elastography, and speckle analysis, to make it more reliable. The acoustic impedance image would play a central role for combining these different data sources. For a more detailed version of this article, we refer the reader to [4]. 
Fig. 2. Acoustic impedance estimation in the overlap area from the 5 simulated US images, transformed in reference coordinate system. Mean of estimations, and in comparison the original CT. (h): Simulation of US image from the global acoustic impedance image (f).

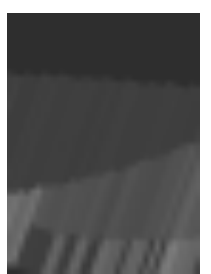

(a) Imp. $-20^{\circ}$

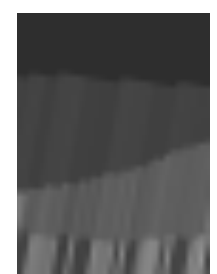

(b) $\operatorname{Imp} .-10^{\circ}$

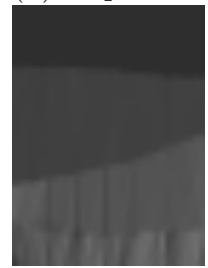

(f) Imp. mean

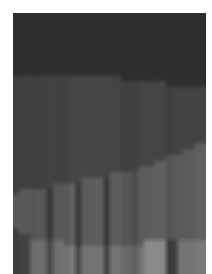

(c) $\operatorname{Imp} .0^{\circ}$

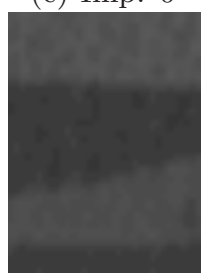

(g) $\mathrm{CT}$

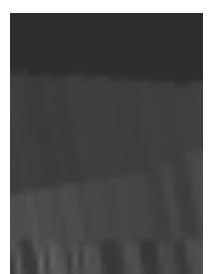

(d) $\operatorname{Imp} \cdot 10^{\circ}$

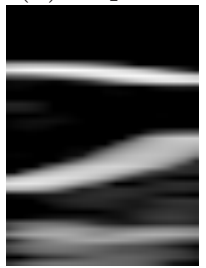

(h) result

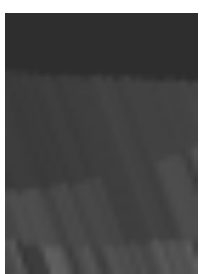

(e) $\operatorname{Imp} \cdot 20^{\circ}$

\section{References}

1. Wilhjelm JE, Jensen MS, Jespersen SK, et al. Visual and quantitative evaluation of selected image combination schemes in ultrasound spatial compound scanning. IEEE Trans Med Imaging. 2004;23(2):181-190.

2. Grau V, Becher H, Noble JA. Registration of multiview real-time 3-D echocardiographic sequences. IEEE Trans Med Imaging. Sept 2007;26(9):1154-1165.

3. Wachinger C, Wein W, Navab N. Three-dimensional ultrasound mosaicing. Proc MICCAI. 2007.

4. Wachinger C, Shams R, Navab N. Estimation of acoustic impedance from multiple ultrasound images with application to spatial compounding. In: Procs IEEE Workshop: Mathematical Methods in Biomedical Image Analysis. Anchorage, Alaska; 2008.

5. Felsberg M, Sommer G. The monogenic signal. IEEE Trans Sign Process. 2001;49(12):3136-3144.

6. Shams R, Hartley R, Navab N. Real-time simulation of medical ultrasound from CT images. Proc MICCAI. 2008. 